\title{
Notes on the peripheral volume of hyperbolic 3-manifolds
}

\author{
Carlo Petronio $\quad$ Michele Tocchet
}

January 29, 2018

\begin{abstract}
We consider hyperbolic 3-manifolds with either non-empty compact geodesic boundary, or some toric cusps, or both. For any such $M$ we analyze what portion of the volume of $M$ can be recovered by inserting in $M$ boundary collars and cusp neighbourhoods with disjoint embedded interiors. Our main result is that this portion can only be maximal in some combinatorially extremal configurations. The techniques we employ are very elementary but the result is in our opinion of some interest. MSC (2010): 57M50 (primary); 57M27 (secondary).
\end{abstract}

The issue of understanding volumes of hyperbolic 3-manifolds has been one of the central themes of geometric topology since the pioneering work of Jorgensen and Thurston, who showed that the set of possible volumes is a well-ordered subset of $\mathbb{R}$ (see, e.g., [2]). In particular, considerable energy has been devoted to identifying the minima of the volume within given classes of manifolds, and the following instances have by now been settled:

- The minimal-volume compact hyperbolic 3-manifolds with non-empty geodesic boundary were proved [12] to be the 8 manifolds that decompose into two truncated regular tetrahedra of dihedral angle $\frac{\pi}{6}$ (these 8 manifolds were first described in [8]);

- The minimal-volume hyperbolic 3-manifolds with toric cusps (but no boundary) were shown [5] to be the figure-eight knot complement and its sibling (these manifolds were first described in [16] and [4]); 
- The minimal-volume closed hyperbolic 3-manifold was very recently proved [9, 10, 11, 14] to be the Weeks manifold (first described in [4] and [13]);

- The two minimal-volume hyperbolic 3-manifolds with two toric cusps (but no boundary) were identified in [1].

Other instances, however, remain open, as that of manifolds with one toric cusp and one compact boundary component.

The general (and very roughly described) idea of the papers quoted so far is to show that an upper or lower bound on the volume implies constraints on the topology of a manifold. Moreover, estimates on the volume of boundary collars or cusp neighbourhoods often play an important role. We also mention that the same ideas appear in other interesting articles, such as [3] and [6]. In this paper we address the following natural question:

- Let $M$ be a hyperbolic 3-dimensional manifold with either non-empty compact geodesic boundary, or some toric cusps, or both. What is the optimal way of inserting in $M$ boundary collars and/or cusp neighbourhoods having disjoint embedded interiors?

Optimality here is of course meant in the sense of volume maximization, collars are defined using the distance, and cusp neighbourhoods are bounded by horospherical cross-sections. We will refer to a boundary collar or cusp neighbourhood using the collective term of peripheral component. Employing very elementary techniques we will prove in this paper that an optimal choice of the peripheral components necessarily occurs in a combinatorially extremal configuration, a fact that we consider to be of some interest.

To state our main results we denote by $v$ the volume function and we establish the convention that, when we mention distinct peripheral components, they always have disjoint and embedded interiors. We then have:

Proposition 0.1. Let $M$ have two peripheral components $P_{1}$ and $P_{2}$. Then $v\left(P_{1}\right)+v\left(P_{2}\right)$ can have a local maximum only if, up to reordering the indices, $P_{1}$ is chosen so that $v\left(P_{1}\right)$ is maximal regardless of $P_{2}$, and then $P_{2}$ is chosen so that $v\left(P_{2}\right)$ is maximal given $P_{1}$.

(When both peripheral components are cusps, or both are collars of boundary components having the same genus, we will also show that one 
must maximize first the component that individually can be made bigger than the other one.)

For the next result we will need to refer to a certain modified volume $\widetilde{v}(P)$ of a peripheral component $P$, that coincides with $v(P)$ when $P$ is a cusp neighbourhood, and will be defined below when $P$ is a boundary collar.

Theorem 0.2. Let $M$ have three peripheral components $P_{1}, P_{2}$, and $P_{3}$. Then $v\left(P_{1}\right)+v\left(P_{2}\right)+v\left(P_{3}\right)$ can have a local maximum only in one of the following configurations:

- Up to reordering the indices, first $P_{1}$ is chosen so that $v\left(P_{1}\right)$ is maximal regardless of $P_{2}$ and $P_{3}$, next $P_{2}$ is chosen so that $v\left(P_{2}\right)$ is maximal given $P_{1}$, and last $P_{3}$ is chosen so that $v\left(P_{3}\right)$ is maximal given $P_{1}, P_{2}$;

- Each of $P_{1}, P_{2}, P_{3}$ is tangent to the other two, and the modified volumes $\widetilde{v}\left(P_{1}\right), \widetilde{v}\left(P_{2}\right), \widetilde{v}\left(P_{3}\right)$ satisfy the strict triangular inequalities.

Moreover, if in the latter case no $P_{j}$ is individually maximal, then the configuration indeed gives a local maximum for $v\left(P_{1}\right)+v\left(P_{2}\right)+v\left(P_{3}\right)$.

Proposition 0.1 and Theorem 0.2 of course have similar flavours, since they both state that a local maximum of the peripheral volume is attained at a combinatorially non-generic configuration, namely one involving individual maximality of one peripheral component or a cycle of tangencies. A punctual analysis similar to that given by Theorem 0.2 is perhaps possible also for four or more peripheral components, but we will refrain from carrying it out. We confine ourselves to the following general result (a more refined version of which will be stated and proved below):

Proposition 0.3. Let $M$ have $n$ peripheral components $P_{1}, \ldots, P_{n}$ such that:

- No $P_{j}$ is such that $v\left(P_{j}\right)$ is maximal regardless of the other $P_{i}$ 's;

- There are fewer than $n$ tangencies between different $P_{j}$ 's.

Then the configuration cannot be a local maximum for $v\left(P_{1}\right)+\ldots+v\left(P_{n}\right)$.

We conclude this introduction with two remarks. First, one could simplify the attempt to partially recover the volume of $M$ by imposing all the collars of the boundary components to have the same width, and all the toric cusps to have the same volume, but our results show that this attempt would 
typically be very inefficient. Second, we explain why we do not consider in this paper the case of manifolds having annular cusps, the reason being that for the boundary components entering such cusps one could not take a collar at all, so the theory would have limited interest.

\section{Preliminaries}

Let us fix for the rest of this article a hyperbolic 3-manifold $M$ with either non-empty compact geodesic boundary, or some toric cusps, or both. We will denote by $\Sigma_{1}, \ldots, \Sigma_{b}$ the components of $\partial M$ and by $T_{1}, \ldots, T_{k}$ the toric boundary components of the compactification of $M$. Moreover for $d>0$ we will indicate by $U_{j}(d)$ the $d$-collar of $\Sigma_{j}$ in $M$, and for $v>0$ by $C_{i}(v)$ the volume- $v$ horospherical cusp neighbourhood at $T_{i}$ in $M$. When certain $U_{j}\left(d_{j}\right)$ 's and $C_{i}\left(v_{i}\right)$ 's are simultaneously considered we will always assume that they have embedded and disjoint interiors. We begin by recalling that each $\Sigma_{j}$ has a well-defined hyperbolic area $\mathcal{A}\left(\Sigma_{j}\right)=-2 \pi \chi\left(\Sigma_{j}\right)$. We then prove the following:

Lemma 1.1. Suppose that $M$ has both geodesic boundary and cusps. Then

the cusp torus $T_{i}$ has a well-defined area $\mathcal{A}_{j}\left(T_{i}\right)=\frac{\mathcal{A}\left(\mathbb{E}^{2} / \Lambda_{i}\right)}{r_{j}^{2}}$ relative to the boundary component $\Sigma_{j}$, where:

- A universal cover $p: \widetilde{M} \rightarrow M$ is chosen with $\widetilde{M}$ being the intersection of a family of hyperbolic half-spaces in the half-space model $\mathbb{E}^{2} \times(0,+\infty)$ of $\mathbb{H}^{3}$, in such a way that with respect to $p$ the torus $T_{i}$ lifts to $\infty$;

- $\Lambda_{i}$ is the lattice acting horizontally on $\mathbb{H}^{3}$ to give the $i$-th cusp of $M$;

- $r_{j}$ is the maximal Euclidean radius of a half-sphere centered at $\mathbb{E}^{2} \times\{0\}$ that bounds $\widetilde{M}$ and projects in $M$ to $\Sigma_{j}$.

Proof. A universal cover as in the statement exists and $r_{j}$ is well-defined because the maximum has to be taken over a $\Lambda_{i}$-equivariant family of halfspheres. Two distinct universal covers as in the statement differ by the composition of a horizontal Euclidean isometry and a dilation in $\mathbb{E}^{2} \times(0,+\infty)$, and the ratio in the statement is preserved by both.

We now start dealing with peripheral volume, by quoting the following formula from [9]: 
Proposition 1.2. $\quad v\left(U_{j}(d)\right)=\frac{\mathcal{A}\left(\Sigma_{j}\right)}{4} \cdot(2 d+\sinh (2 d))$.

Before proceeding, we define the modified volume $\widetilde{v}$ for a boundary collar $U_{j}(d)$ by setting

$$
\widetilde{v}\left(U_{j}(d)\right)=\frac{\mathcal{A}\left(\Sigma_{j}\right)}{4} \cdot(1+\cosh (2 d)) .
$$

Note that $\widetilde{v}\left(U_{j}(d)\right)$ is a strictly increasing function of $v\left(U_{j}(d)\right)$, but one cannot describe the function explicitly.

The next result will be the core of our arguments. It describes how a peripheral component changes when it varies subject to the condition of staying tangent to another one that is also varying.

\section{Proposition 1.3.}

- If $C_{i_{1}}\left(v_{1}\right)$ and $C_{i_{2}}\left(v_{2}\right)$ vary while remaining tangent to each other, then the product $v_{1} \cdot v_{2}$ remains constant;

- If $U_{j}(d)$ and $C_{i}(v)$ vary while remaining tangent to each other then $v=\frac{\mathcal{A}_{j}\left(T_{i}\right)}{2} \cdot e^{-2 d}$

- If $U_{j_{1}}\left(d_{1}\right)$ and $U_{j_{2}}\left(d_{2}\right)$ vary while remaining tangent to each other, then the sum $d_{1}+d_{2}$ remains constant.

Proof. Let us prove the first statement. Suppose that in some universal cover contained in $\mathbb{H}^{3}=\mathbb{E}^{2} \times(0,+\infty)$ the cusp $C_{i_{1}}\left(v_{1}\right)$ is the quotient of the horoball $\mathbb{E}^{2} \times\left[z_{i_{1}},+\infty\right)$ acted on horizontally by a lattice $\Lambda_{i_{1}}$. If the cusp changes so that in $M$ its boundary moves of some small distance $d \in \mathbb{R}$ (with $d<0$ meaning that the cusp is shrinking) then it becomes the quotient under $\Lambda_{i_{1}}$ of $\mathbb{E}^{2} \times[z,+\infty)$ with

$$
\int_{z}^{z_{i_{1}}} \frac{1}{t} \mathrm{~d} t=d
$$

whence $z=z_{i_{1}} e^{-d}$. The cusp volume $v_{1}$ then changes from

$$
\mathcal{A}\left(\mathbb{E}^{2} / \Lambda_{i_{1}}\right) \cdot \int_{z_{i_{1}}}^{+\infty} \frac{1}{t^{3}} \mathrm{~d} t=\frac{\mathcal{A}\left(\mathbb{E}^{2} / \Lambda_{i_{1}}\right)}{2} \cdot z_{i_{1}}^{-2}
$$


to $\frac{\mathcal{A}\left(\mathbb{E}^{2} / \Lambda_{i_{1}}\right)}{2} \cdot z^{-2}$, namely it changes by a factor $e^{2 d}$. During a simultaneous variation of $v_{1}$ and $v_{2}$ with $C_{i_{1}}\left(v_{1}\right)$ and $C_{i_{2}}\left(v_{2}\right)$ remaining tangent to each other, the boundary of $C_{i_{2}}\left(v_{2}\right)$ moves by a distance $-d$. The calculations already carried out show that then its volume varies by a factor $e^{-2 d}$, and the conclusion follows.

Turning to the second statement, we choose a universal cover of $M$ with $C_{i}(v)$ lifting to some horoball $\mathbb{E}^{2} \times[z,+\infty)$ in $\mathbb{E}^{2} \times(0,+\infty)$. With $r_{j}$ being as in the definition of $\mathcal{A}_{j}\left(T_{i}\right)$, the condition that $U_{j}(d)$ is tangent to $C_{i}(V)$ implies that

$$
\int_{r_{j}}^{z} \frac{1}{t} \mathrm{~d} t=d
$$

whence $z=r_{j} e^{d}$ and

$$
v=\frac{\mathcal{A}\left(\mathbb{E}^{2} / \Lambda_{i_{1}}\right)}{2 r_{j}^{2}} \cdot e^{-2 d}=\frac{\mathcal{A}_{j}\left(T_{i}\right)}{2} \cdot e^{-2 d} .
$$

This proves the desired formula. The third statement is obvious.

\section{Two peripheral components and the general case}

We will establish here the two propositions stated in the introduction. For the sake of conciseness let us say that a a peripheral component $P$ is maximal if $v(P)$ is maximal regardless of the other peripheral components, i.e., if $\partial P$ is tangent either to itself or to $\partial M$.

Proof of Proposition 0.1. We must show that $v\left(P_{1}\right)+v\left(P_{2}\right)$ cannot have a local maximum unless, up to switching indices, $P_{1}$ is maximal and $P_{2}$ is either maximal or tangent to $P_{1}$. If one of $P_{1}$ or $P_{2}$ is not maximal or tangent to the other one, of course $v\left(P_{1}\right)+v\left(P_{2}\right)$ cannot be locally maximal. We are then left to exclude only the situation where $P_{1}$ and $P_{2}$ are tangent to each other but neither of them is tangent to itself or to the boundary. To this configuration we can locally apply Proposition [1.3, Depending on whether $P_{1}$ and $P_{2}$ are both cusps, a cusp and a collar, or two collars, the total volume 
$V=v\left(P_{1}\right)+v\left(P_{2}\right)$ with respect to an appropriate parameter is given by

$$
\begin{aligned}
C_{1}\left(v_{1}\right) \cup C_{2}\left(v_{2}\right): & V\left(v_{1}\right) & =v_{1}+\frac{v_{0}^{2}}{v_{1}} \quad \text { for some } v_{0}>0, \\
U_{1}\left(d_{1}\right) \cup C_{1}\left(v_{1}\right): & V\left(d_{1}\right) & =\frac{\mathcal{A}\left(\Sigma_{1}\right)}{4} \cdot\left(2 d_{1}+\sinh \left(2 d_{1}\right)\right)+\frac{\mathcal{A}_{1}\left(T_{1}\right)}{2} \cdot e^{-2 d_{1}}, \\
U_{1}\left(d_{1}\right) \cup U_{2}\left(d_{2}\right): & V\left(d_{1}\right) & =\frac{\mathcal{A}\left(\Sigma_{1}\right)}{4} \cdot\left(2 d_{1}+\sinh \left(2 d_{1}\right)\right) \\
& +\frac{\mathcal{A}\left(\Sigma_{2}\right)}{4} \cdot\left(2\left(2 d_{0}-d_{1}\right)+\sinh \left(2\left(2 d_{0}-d_{1}\right)\right)\right) & \text { for some } d_{0}>0 .
\end{aligned}
$$

Since in all cases $V$ is a convex function and our starting point is in the interior of the domain of definition of $V$, the conclusion follows.

In two special cases for the types of the two peripheral components we have the following improvement on Proposition 0.1, also announced above:

Proposition 2.1. Suppose that $M$ has either two cusps and no geodesic boundary or two geodesic boundary components of the same genus and no cusps. For $j=1,2$ let $v_{j}^{\max }$ be the maximal volume that the $j$-th peripheral component $P_{j}$ can attain regardless of the other one. If $v_{1}^{\max } \geqslant v_{2}^{\max }$ then the maximum of $v\left(P_{1}\right)+v\left(P_{2}\right)$ is attained by maximizing first $P_{1}$ and then $P_{2}$ given $P_{1}$.

Proof. We start with the case of two cusps. Taking $v_{1}$ as a variable to parameterize $C_{1}\left(v_{1}\right) \cup C_{2}\left(v_{2}\right)$ in tangency position, we have $v_{2}=\frac{v_{0}^{2}}{v_{1}}$ for some $v_{0}>0$, and $v_{1}$ varies in $\left[v_{1}^{\min }, v_{1}^{\max }\right]$ with $v_{1}^{\min }=\frac{v_{0}^{2}}{v_{2}^{\max }}$. We must maximize on $\left[v_{1}^{\min }, v_{1}^{\max }\right]$ the convex function $v_{1} \mapsto v_{1}+\frac{v_{0}^{2}}{v_{1}}$, that attains its minimum at $v_{1}=v_{0}$. We may now have $v_{1}^{\min }>v_{0}$ or $v_{1}^{\text {min }} \leqslant v_{0}$. In the former case of course the maximum of $v_{1}+\frac{v_{0}^{2}}{v_{1}}$ is attained at $v_{1}^{\max }$. In the latter case we have $v_{2}^{\max }=\frac{v_{0}^{2}}{v_{1}^{\min }} \geqslant v_{0}$, but $v \mapsto v+\frac{v_{0}^{2}}{v}$ is increasing on $\left[v_{0},+\infty\right)$, therefore the inequalities $v_{1}^{\max } \geqslant v_{2}^{\max } \geqslant v_{0}$ imply

$$
v_{1}^{\max }+\frac{v_{0}^{2}}{v_{1}^{\max }} \geqslant v_{2}^{\max }+\frac{v_{0}^{2}}{v_{2}^{\max }}=\frac{v_{0}^{2}}{v_{1}^{\min }}+v_{1}^{\min }
$$

and the conclusion follows.

For two boundary components of the same genus $g$ the argument is similar. Let $d_{j}^{\max }$ be the maximal $d_{j}$ such that $U_{j}\left(d_{j}\right)$ has embedded interior, and 
let $d_{i}^{\text {min }}$ for $\{i, j\}=\{1,2\}$ be the maximal $d_{i}$ such that $U_{i}\left(d_{i}\right)$ has embedded interior in $M \backslash U_{j}\left(d_{j}^{\max }\right)$. Note that $d_{1}^{\max }+d_{2}^{\min }=d_{1}^{\min }+d_{2}^{\max }$, and denote by $2 d_{0}$ this quantity. Since $v_{j}^{\max }=\pi(g-1)\left(2 d_{j}^{\max }+\sinh \left(2 d_{j}^{\max }\right)\right)$ we have $d_{1}^{\max } \geqslant d_{2}^{\max }$. Using $d_{1}$ as a deformation parameter we must now maximize on $\left[d_{1}^{\min }, d_{1}^{\max }\right]$ the convex function

$$
f\left(d_{1}\right)=\pi(g-1)\left(2 d_{1}+\sinh \left(2 d_{1}\right)+2\left(2 d_{0}-d_{1}\right)+\sinh \left(2\left(2 d_{0}-d_{1}\right)\right)\right),
$$

that attains its minimum at $d_{0}$. If $d_{1}^{\text {min }} \geqslant d_{0}$ the maximum is of course attained at $d_{1}^{\max }$. If $d_{1}^{\min }<d_{0}$ we have $d_{2}^{\max }>d_{0}$, but we also know that $d_{1}^{\max } \geqslant d_{2}^{\max }$, whence

$$
f\left(d_{1}^{\max }\right) \geqslant f\left(d_{2}^{\max }\right)=f\left(d_{1}^{\min }\right)
$$

and the proof is complete.

We conclude this section by proving a result that easily implies Proposition 0.3 ,

Proposition 2.2. Let $M$ have $n$ peripheral components $P_{1}, \ldots, P_{n}$ and construct a graph with vertices $P_{1}, \ldots, P_{n}$ and edges joining peripheral components that are tangent to each other. Suppose that this graph has a connected component $\Gamma$ such that:

- $\Gamma$ is a tree;

- No vertex $P_{j}$ of $\Gamma$ is maximal.

Then the configuration cannot be a local maximum for $v\left(P_{1}\right)+\ldots+v\left(P_{n}\right)$.

Proof. Under the stated assumptions we can locally deform the peripheral components corresponding to the vertices in $\Gamma$ using one parameter that can be both increased and decreased. If in $\Gamma$ there are only cusps and no boundary components, choosing $v_{1}$ as a deformation parameter we see that each other $v_{i}$ in $\Gamma$ varies either as $c_{i} \cdot v_{1}$ or as $\frac{c_{i}}{v_{1}}$ for some $c_{i}>0$, which implies that the sum of all the volumes of the peripheral components in $\Gamma$ is a convex function of $v_{1}$, whence the conclusion.

Suppose then that in $\Gamma$ there exists at least one boundary collar component and choose $d_{1}$ as a deformation parameter. We then claim that each $d_{j}$ in $\Gamma$ varies as either $c_{j}+d_{1}$ or as $c_{j}-d_{1}$ for some $c_{j} \in \mathbb{R}$, and each $v_{i}$ in $\Gamma$ varies as either $c_{i} \cdot e^{-2 d_{1}}$ or as $c_{i} \cdot e^{2 d_{1}}$ for some $c_{i}>0$. This can be 
easily checked using Proposition 1.3 and induction on the number of edges in $\Gamma$ one needs to travel through in passing from $U_{1}\left(d_{1}\right)$ to $U_{j}\left(d_{j}\right)$ or $C_{i}\left(v_{i}\right)$. Since $v\left(U_{j}\left(d_{j}\right)\right)$ is a convex function of $d_{j}$ we deduce that the sum of the volumes of the peripheral components in $\Gamma$ is a convex function of $d_{1}$, and the proposition is proved.

Experimental facts A census was carried out in [7] of all the 5,192 hyperbolic 3-manifolds with non-empty compact geodesic boundary that can be triangulated using up to 4 tetrahedra. It turns out that the geodesic boundary is always connected, and that there is one cusp for 31 manifolds and two cusps for one manifold. We were now able to check [15] that for all the 32 relevant cases the largest peripheral volume is obtained by maximizing first the boundary collar and then the cusp neighbourhood(s, that both become tangent to the boundary collar when there are two of them, before becoming tangent themselves or to each other). ).

\section{Three peripheral components}

This section is devoted to the proof of Theorem 0.2. We already know from Proposition 0.3 that at a local maximum for $v\left(P_{1}\right)+v\left(P_{2}\right)+v\left(P_{3}\right)$ either some $P_{j}$ is maximal or each $P_{j}$ is tangent to each other $P_{i}$. In the former case, suppose that $P_{1}$ is maximal. If $P_{2}$ or $P_{3}$ is maximal given $P_{1}$ then up to switching $P_{2}$ and $P_{3}$ we have a configuration as described in the first item of the statement. Otherwise $P_{2}$ and $P_{3}$ are tangent to each other but not to themselves or to $P_{1}$, and the argument given in the proof of Proposition 0.1 shows that the configuration cannot locally maximize the volume.

We are left to deal with the configuration in which each $P_{j}$ is tangent to each other $P_{i}$ but it is not individually maximal. This implies that each $P_{j}$ is not tangent to itself and that it has positive width if it is of collar type (otherwise some other $P_{i}$ would be maximal). In this case the local deformation we can perform is as follows:

- We can inflate $P_{1}$ and shrink $P_{2}$ and $P_{3}$ so that they stay tangent to $P_{1}$, with shapes varying as described in Proposition 1.3,

- We can shrink $P_{1}$, in which case we can further deform $P_{2}$ and $P_{3}$ in such a way that they stay tangent to each other. But thanks to the argument showing Proposition 0.1 we know that along this deformation 
we cannot have a local maximum except at the extrema, namely when either $P_{2}$ or $P_{3}$ is tangent to $P_{1}$.

To analyze exactly how the volume behaves under this deformation we need to distinguish according to the types of $P_{1}, P_{2}, P_{3}$, noting that our choice of $P_{1}$ in the above description of the deformation was an arbitrary one. The argument is similar in all four cases, with complications increasing (and amount of details we supply decreasing) as the number of boundary components grows.

Case I: Three Cusps Let $C_{1}\left(v_{1}^{(0)}\right), C_{2}\left(v_{2}^{(0)}\right), C_{3}\left(v_{3}^{(0)}\right)$ be the initial cusps with indices chosen so that $v_{2}^{(0)} \geqslant v_{3}^{(0)}$. We then let $v_{1}$ vary in a neighbourhood of $v_{1}^{(0)}$ and note that, according to the above description of the deformation, for $v_{1} \leqslant v_{1}^{(0)}$ the total deformed volume is given by $v_{1}$ plus

$$
\max \left\{\frac{v_{1}^{(0)} \cdot v_{2}^{(0)}}{v_{1}}+\frac{v_{3}^{(0)}}{v_{1}^{(0)}} \cdot v_{1}, \frac{v_{2}^{(0)}}{v_{1}^{(0)}} \cdot v_{1}+\frac{v_{1}^{(0)} \cdot v_{3}^{(0)}}{v_{1}}\right\}
$$

but the assumption $v_{2}^{(0)} \geqslant v_{3}^{(0)}$ readily implies that the maximum is given by the first expression. Therefore near $v_{1}^{(0)}$ the total deformed volume is

$$
V\left(v_{1}\right)=\left\{\begin{array}{l}
v_{1}+\frac{v_{1}^{(0)} \cdot v_{2}^{(0)}}{v_{1}}+\frac{v_{3}^{(0)}}{v_{1}^{(0)}} \cdot v_{1} \quad \text { for } \quad v_{1} \leqslant v_{1}^{(0)} \\
v_{1}+\frac{v_{1}^{(0)} \cdot v_{2}^{(0)}}{v_{1}}+\frac{v_{1}^{(0)} \cdot v_{3}^{(0)}}{v_{1}} \text { for } \quad v_{1} \geqslant v_{1}^{(0)} .
\end{array}\right.
$$

We now have

$$
V_{-}^{\prime}\left(v_{1}^{(0)}\right)=1-\frac{v_{2}^{(0)}}{v_{1}^{(0)}}+\frac{v_{3}^{(0)}}{v_{1}^{(0)}}, \quad V_{+}^{\prime}\left(v_{1}^{(0)}\right)=1-\frac{v_{2}^{(0)}}{v_{1}^{(0)}}-\frac{v_{3}^{(0)}}{v_{1}^{(0)}}
$$

and we note that under the assumption $v_{2}^{(0)} \geqslant v_{3}^{(0)}$ the strict triangular inequalities for $v_{1}^{(0)}, v_{2}^{(0)}, v_{3}^{(0)}$ read as

$$
v_{2}^{(0)}-v_{3}^{(0)}<v_{1}^{(0)}<v_{2}^{(0)}+v_{3}^{(0)},
$$

therefore they are equivalent to the conditions

$$
V_{+}^{\prime}\left(v_{1}^{(0)}\right)<0<V_{-}^{\prime}\left(v_{1}^{(0)}\right)
$$


so they imply that $V$ has a local maximum at $v_{1}^{(0)}$. We must show that, conversely, if one of the strict triangular inequalities is violated then $V$ does not have a local maximum at $v_{1}^{(0)}$, which is because in this case we have either $V_{-}^{\prime}\left(v_{1}^{(0)}\right) \leqslant 0$ or $V_{+}^{\prime}\left(v_{1}^{(0)}\right) \geqslant 0$, but $V_{-}^{\prime \prime}\left(v_{1}^{(0)}\right)>0$ and $V_{+}^{\prime \prime}\left(v_{1}^{(0)}\right)>0$.

CASE II: One BOUndary COMPONEnT AND two Cusps We denote by $\Sigma$ the boundary component and for $i=2,3$ by $\mathcal{A}\left(T_{i}\right)$ the areas relative to $\Sigma$ of the tori $T_{2}$ and $T_{3}$, with indices chosen so that $\mathcal{A}\left(T_{2}\right) \geqslant \mathcal{A}\left(T_{3}\right)$. Suppose that the initial peripheral components are $U_{1}\left(d_{1}^{(0)}\right), C_{2}\left(v_{2}^{(0)}\right), C_{3}\left(v_{3}^{(0)}\right)$, with volumes

$$
v_{1}^{(0)}=\frac{\mathcal{A}(\Sigma)}{4} \cdot\left(2 d_{1}^{(0)}+\sinh \left(2 d_{1}^{(0)}\right)\right), \quad v_{i}^{(0)}=\frac{\mathcal{A}\left(T_{i}\right)}{2} \cdot e^{-2 d_{1}^{(0)}}
$$

(therefore $v_{2}^{(0)} \geqslant v_{3}^{(0)}$ by our choice of the indices). Using $d_{1}$ to parameterize the deformation we have for $d_{1}<d_{1}^{(0)}$ that the deformed total volume is given by $\frac{\mathcal{A}(\Sigma)}{4} \cdot\left(2 d_{1}+\sinh \left(2 d_{1}\right)\right)$ plus

$$
\begin{aligned}
\max \left\{\frac{\mathcal{A}\left(T_{2}\right)}{2} \cdot e^{-2 d_{1}}+\right. & \frac{\mathcal{A}\left(T_{3}\right)}{2} \cdot e^{-2\left(2 d_{1}^{(0)}-d_{1}\right)}, \\
& \left.\frac{\mathcal{A}\left(T_{2}\right)}{2} \cdot e^{-2\left(2 d_{1}^{(0)}-d_{1}\right)}+\frac{\mathcal{A}\left(T_{3}\right)}{2} \cdot e^{-2 d_{1}}\right\}
\end{aligned}
$$

and the first expression prevails thanks to the assumption $\mathcal{A}\left(T_{2}\right) \geqslant \mathcal{A}\left(T_{3}\right)$. The deformed total volume is therefore

$$
V\left(d_{1}\right)=\left\{\begin{array}{rr}
\frac{\mathcal{A}(\Sigma)}{4} \cdot\left(2 d_{1}+\sinh \left(2 d_{1}\right)\right)+\frac{\mathcal{A}\left(T_{2}\right)}{2} \cdot e^{-2 d_{1}}+\frac{\mathcal{A}\left(T_{3}\right)}{2} \cdot e^{2 d_{1}-4 d_{1}^{(0)}} & \text { for } \quad d_{1} \leqslant d_{1}^{(0)} \\
\frac{\mathcal{A}(\Sigma)}{4} \cdot\left(2 d_{1}+\sinh \left(2 d_{1}\right)\right)+\frac{\mathcal{A}\left(T_{2}\right)}{2} \cdot e^{-2 d_{1}}+\frac{\mathcal{A}\left(T_{3}\right)}{2} \cdot e^{-2 d_{1}} & \text { for } d_{1} \geqslant d_{1}^{(0)}
\end{array}\right.
$$

and we easily have

$$
V_{-}^{\prime}\left(d_{1}^{(0)}\right)=2\left(\widetilde{v}_{1}^{(0)}-v_{2}^{(0)}+v_{3}^{(0)}\right), \quad V_{+}^{\prime}\left(d_{1}^{(0)}\right)=2\left(\widetilde{v}_{1}^{(0)}-v_{2}^{(0)}-v_{3}^{(0)}\right) .
$$

Since $V_{ \pm}^{\prime \prime}\left(d_{1}^{(0)}\right)>0$, the conclusion follows precisely as in Case I. 
CASE III: Two BOUndary COMPOnEnts And OnE CUSP Let the peripheral components be $C_{1}\left(v_{1}^{(0)}\right), U_{2}\left(d_{2}^{(0)}\right), U_{3}\left(d_{3}^{(0)}\right)$, whence

$$
v_{1}^{(0)}=\frac{\mathcal{A}_{2}\left(T_{1}\right)}{2} \cdot e^{-2 d_{2}^{(0)}}=\frac{\mathcal{A}_{3}\left(T_{1}\right)}{2} \cdot e^{-2 d_{3}^{(0)}} .
$$

For the sake of brevity we now set $f_{j}(t)=\frac{\mathcal{A}\left(\Sigma_{j}\right)}{4}(2 t+\sinh (2 t))$, so that $v\left(U_{j}\left(d_{j}\right)\right)=f_{j}\left(d_{j}\right)$, and $f_{j}^{\prime}\left(d_{j}^{(0)}\right)=2 \widetilde{v}_{j}^{(0)}$. We now choose indices so that $\widetilde{v}_{2}^{(0)} \geqslant \widetilde{v}_{3}^{(0)}$. We deform the configuration using the parameter $v_{1}$, to do which we define the functions

$$
d_{j}\left(v_{1}\right)=-\frac{1}{2} \log \frac{2 v_{1}}{\mathcal{A}_{j}\left(T_{1}\right)},
$$

noting that for $v_{1} \geqslant v_{1}^{(0)}$ the peripheral configuration is given by

$$
C_{1}\left(v_{1}\right), U_{2}\left(d_{2}\left(v_{1}\right)\right), U_{3}\left(d_{3}\left(v_{1}\right)\right) \text {. }
$$

For $v_{1}<v_{1}^{(0)}$, on the contrary, we have one of the following:

- $d_{2}=d_{2}\left(v_{1}\right)$ and $d_{3}=d_{3}^{(0)}+d_{2}^{(0)}-d_{2}\left(v_{1}\right)$,

- $d_{3}=d_{3}\left(v_{1}\right)$ and $d_{2}=d_{2}^{(0)}+d_{3}^{(0)}-d_{3}\left(v_{1}\right)$.

The total deformed volume for $v_{1} \leqslant v_{1}^{(0)}$ is then $v_{1}$ plus

$$
\begin{aligned}
\max \left\{f_{2}\left(d_{2}\left(v_{1}\right)\right)+\right. & f_{3}\left(d_{3}^{(0)}+d_{2}^{(0)}-d_{2}\left(v_{1}\right)\right), \\
& \left.f_{2}\left(d_{2}^{(0)}+d_{3}^{(0)}-d_{3}\left(v_{1}\right)\right)+f_{3}\left(d_{3}\left(v_{1}\right)\right)\right\}
\end{aligned}
$$

and the first expression prevails thanks to our assumption, because at the point $v_{1}=v_{1}^{(0)}$ it has the same value as the second expression but smaller first derivative. Therefore

$$
V\left(v_{1}\right)= \begin{cases}v_{1}+f_{2}\left(d_{2}\left(v_{1}\right)\right)+f_{3}\left(d_{3}^{(0)}+d_{2}^{(0)}-d_{2}\left(v_{1}\right)\right) & \text { for } v_{1} \leqslant v_{1}^{(0)} \\ v_{1}+f_{2}\left(d_{2}\left(v_{1}\right)\right)+f_{3}\left(d_{3}\left(v_{1}\right)\right) & \text { for } v_{1} \geqslant v_{1}^{(0)}\end{cases}
$$

whence

$$
V_{-}^{\prime}\left(v_{1}^{(0)}\right)=1-\frac{\widetilde{v}_{2}^{(0)}}{v_{1}^{(0)}}+\frac{\widetilde{v}_{3}^{(0)}}{v_{1}^{(0)}}, \quad V_{+}^{\prime}\left(v_{1}^{(0)}\right)=1-\frac{\widetilde{v}_{2}^{(0)}}{v_{1}^{(0)}}-\frac{\widetilde{v}_{3}^{(0)}}{v_{1}^{(0)}}
$$


and precisely as in the previous two cases we conclude that we have a local maximum if and only if $v_{1}^{(0)}, \widetilde{v}_{2}^{(0)}, \widetilde{v}_{3}^{(0)}$ satisfy the strict triangular inequalities.

CASE IV: Three BOUndary COMPONEnTs Let the boundary components be $\Sigma_{1}, \Sigma_{2}, \Sigma_{3}$ and the initial configuration be $U_{1}\left(d_{1}^{(0)}\right), U_{2}\left(d_{2}^{(0)}\right), U_{3}\left(d_{3}^{(0)}\right)$ with $\widetilde{v}_{2}^{(0)} \geqslant \widetilde{v}_{3}^{(0)}$. Setting

$$
f_{j}(t)=\frac{\mathcal{A}\left(\Sigma_{j}\right)}{4}(2 t+\sinh (2 t))
$$

and using $d_{1}$ to parameterize the deformation we have that the deformed volume is given by

$$
V\left(d_{1}\right)=\left\{\begin{array}{cc}
f_{1}\left(d_{1}\right)+f_{2}\left(d_{2}^{(0)}+d_{1}^{(0)}-d_{1}\right)+f_{3}\left(d_{3}^{(0)}-d_{1}^{(0)}+d_{1}\right) \\
\text { for } d_{1} \leqslant d_{1}^{(0)} \\
f_{1}\left(d_{1}\right)+f_{2}\left(d_{2}^{(0)}+d_{1}^{(0)}-d_{1}\right)+f_{3}\left(d_{3}^{(0)}+d_{1}^{(0)}-d_{1}\right) \\
& \text { for } d_{1} \geqslant d_{1}^{(0)} .
\end{array}\right.
$$

This gives

$$
V_{-}^{\prime}\left(d_{1}^{(0)}\right)=2\left(\widetilde{v}_{1}^{(0)}-\widetilde{v}_{2}^{(0)}+\widetilde{v}_{3}^{(0)}\right), \quad V_{+}^{\prime}\left(d_{1}^{(0)}\right)=2\left(\widetilde{v}_{1}^{(0)}-\widetilde{v}_{2}^{(0)}-\widetilde{v}_{3}^{(0)}\right)
$$

and the conclusion is once again the same.

\section{References}

[1] I. Agol, The minimal volume orientable hyperbolic 2-cusped 3manifolds, Proc. Amer. Math. Soc. 138 (2010), 3723-3732.

[2] R. Benedetti, C. Petronio, "Lectures on Hyperbolic Geometry," Springer (1992).

[3] M. BRIdgeman, J. KAHn, Hyperbolic volume of manifolds with geodesic boundary and orthospectra, Geom. Funct. Anal. 20 (2010), 1210-1230.

[4] P. J. Callahan, M. V. Hildebrandt, J. R. Weeks, A census of cusped hyperbolic 3-manifolds, Math. Comp. 68 (1999), 321-332. 
[5] C. CaO, G. R. Meyerhoff, The orientable cusped hyperbolic 3manifolds of minimum volume, Invent. Math. 146 (2001) 451-478.

[6] J. DeBlois, P. B. Shalen, Volume and topology of bounded and closed hyperbolic 3-manifolds, Comm. Anal. Geom. 17 (2009), 797-849.

[7] R. Frigerio, B. Martelli, C. Petronio, Small hyperbolic 3manifolds with geodesic boundary, Experiment. Math. 13 (2004), 171184.

[8] M. FuJII, Hyperbolic 3-manifolds with totally geodesic boundary which are decomposed into hyperbolic truncated tetrahedra, Tokyo J. Math. 13 (1990), No.2 353-373.

[9] D. Gabai, G. R. Meyerhoff, P. Milley, Mom technology and volumes of hyperbolic 3-manifolds, Comment. Math. Helv. 86 (2011), 145-188.

[10] D. Gabai, G. R. Meyerhoff, P. Milley, Minimum volume cusped hyperbolic three-manifolds, J. Amer. Math. Soc. 22 (2009), 1157-1215.

[11] D. Gabai, G. R. Meyerhoff, P. Milley, Mom technology and hyperbolic 3-manifolds, from: "In the tradition of Ahlfors-Bers, V" (W. Bonk, J. Gilman, H. Masur, Y. Minsky, M. Wolf, editors), Contemp. Math. 510, Amer. Math. Soc., Providence, RI (2010), 84-107.

[12] S. Kojima, Y. Мiуамото, The smallest hyperbolic 3-manifolds with totally geodesic boundary, J. Differential Geom. 34 (1991), 175-192.

[13] S. V. Matveev, A. T. Fomenko, Constant energy surfaces of Hamiltonian systems, enumeration of three-dimensional manifolds in increasing order of complexity, and computation of volumes of closed hyperbolic manifolds, Russ. Math. Surv. 43 (1988), no. 1324.

[14] P. Milley, Minimum volume hyperbolic 3-manifolds, J. Topol. (1) 2 (2009), 181-192.

[15] M. Tocchet, "'Generalized Mom-structures and volume estimates on hyperbolic 3-manifolds with geodesic boundary and toric cusps," PhD thesis, "Sapienza" Università di Roma, 2012. 
[16] W. P. Thurston, "The Geometry and Topology of 3-Manifolds," Princeton University Press (1978).

Dipartimento di Matematica Applicata

Università di Pisa

Via Filippo Buonarroti, 1C

56127 PISA - Italy

petronio@dm.unipi.it

Dipartimento di Matematica

"Sapienza" Università di Roma

Piazzale Aldo Moro, 5

00185 ROMA - Italy

tocchet@mat.uniroma1.it 